This item was submitted to Loughborough's Research Repository by the author.

Items in Figshare are protected by copyright, with all rights reserved, unless otherwise indicated.

\title{
Material property models for polyethylene-based conductive blends suitable for PEM fuel cell bipolar plates
}

PLEASE CITE THE PUBLISHED VERSION

http://dx.doi.org/10.1177/1464420714550192

\section{PUBLISHER}

(C) Institution of Mechanical Engineers

\section{VERSION}

SMUR (Submitted Manuscript Under Review)

\section{PUBLISHER STATEMENT}

This work is made available according to the conditions of the Creative Commons Attribution-NonCommercialNoDerivatives 4.0 International (CC BY-NC-ND 4.0) licence. Full details of this licence are available at: https://creativecommons.org/licenses/by-nc-nd/4.0/

\section{LICENCE}

CC BY-NC-ND 4.0

\section{REPOSITORY RECORD}

Greenwood, Paul S., R.H. Thring, and Rui Chen. 2016. "Material Property Models for Polyethylene-based Conductive Blends Suitable for PEM Fuel Cell Bipolar Plates". figshare. https://hdl.handle.net/2134/21009. 


\section{Material property models for polyethylene based conductive blends suitable for PEM fuel cell bipolar plates.}

\begin{tabular}{|r|l|}
\hline Journal: & Part L: Journal of Materials: Design and Applications \\
\hline Manuscript ID: & JMDA-13-0077.R1 \\
\hline Manuscript Type: & Original article \\
\hline Date Submitted by the Author: & $\mathrm{n}$ /a \\
\hline Complete List of Authors: & $\begin{array}{l}\text { Greenwood, Paul; Loughborough University, } \\
\text { Thring, Rob; University of Loughborough } \\
\text { Chen, R; University of Loughborough }\end{array}$ \\
\hline Keywords: & $\begin{array}{l}\text { Materials Modelling, Bipolar Plate, Conductivity, Modulus, Carbon Black, } \\
\text { Graphite, Magnetite, Fuel Cell }\end{array}$ \\
\hline Abstract: & $\begin{array}{l}\text { Electrical and thermal conductivity models from Mamunya et al and } \\
\text { Kerner's equation for elastic and flexural modulus have been applied to } \\
\text { experimental data to assess model accuracies. Experimental data was } \\
\text { gained from a previous study where polyethylene and carbon black, } \\
\text { graphite and magnetite composites produced by injection and compression } \\
\text { moulding were tested. The electrical conductivity modelling gave accurate } \\
\text { fits to the data, though available data was limited and the thermal } \\
\text { conductivity modelling produced good fits with R2 values greater than } \\
\text { 0.93. The electrical and thermal model exponents were tuned for best fit } \\
\text { and used to compare the modelling results with the literature and gain } \\
\text { information about conduction mechanisms such as tunnelling and links, } \\
\text { nodes and blobs and variations in local filler concentrations. A modified } \\
\text { Kerner's equation to effectively allow for filler type and variations in } \\
\text { composite processing was used to improve upon the original equation } \\
\text { which modelled modulus behaviour based on the pure matrix. The new } \\
\text { equation proved to be a better predictor of elastic modulus than flexural } \\
\text { modulus. }\end{array}$ \\
\hline & \begin{tabular}{l}
$\mid$ \\
\hline
\end{tabular} \\
\hline
\end{tabular}




\title{
Material property models for polyethylene based conductive blends suitable for PEM fuel cell bipolar plates.
}

\author{
Greenwood P, Thring R.H, Chen R.
}

\begin{abstract}
Electrical and thermal conductivity models from Mamunya et al and Kerner's equation for elastic and flexural modulus have been applied to experimental data to assess model accuracies. Experimental data was gained from a previous study where polyethylene and carbon black, graphite and magnetite composites produced by injection and compression moulding were tested. The electrical conductivity modelling gave accurate fits to the data, though available data was limited and the thermal conductivity modelling produced good fits with $\mathrm{R}^{2}$ values greater than 0.93 . The electrical and thermal model exponents were tuned for best fit and used to compare the modelling results with the literature and gain information about conduction mechanisms such as tunnelling and links, nodes and blobs and variations in local filler concentrations. A modified Kerner's equation to effectively allow for filler type and variations in composite processing was used to improve upon the original equation which modelled modulus behaviour based on the pure matrix. The new equation proved to be a better predictor of elastic modulus than flexural modulus.
\end{abstract}

Keywords: Materials Modelling, Bipolar Plate, Conductivity, Modulus, Carbon Black, Graphite, Magnetite, Fuel Cell. 


\section{Introduction}

Electrical and thermal conductivity and the tensile and flexural strength results of high density polyethylene (HDPE) blends with carbon black, graphite and magnetite fillers presented in a previous study ${ }^{[1]}$ have been used here to assess and validate models used to predict such properties.

To understand these properties of the composites and the mechanisms that contribute to them, theories have been developed to model the behaviour of the materials with varying filler content. In this way the models can help tailor / optimise the composite design in order to better fit the application and reduce costs. As stated by Lux ${ }^{[2]}$, the following parameters should be taken into account for all electrical conductivity models.

- Size and geometry of the filler.

- Quantity and distribution of the filler.

- Interaction between conductive and insulating components.

- Preparation method of the mixtures.

This would also be true for thermal conductivity models due to the similar physical conduction requirements as electrical conductivity and also true for mechanical models where the above factors would affect fracture behaviour.

The size and geometry of the filler would affect contact surface area between particles and at the filler-matrix interfaces therefore determining the level of conduction for electrical and thermal properties and adhesion for mechanical properties. For spherical particles, lower

percolation threshold is obtained with smaller particle size ${ }^{[3,4]}$. Similarly the quantity and 
distribution of the filler would increase or decrease the probability of such contact between particles. The interaction between filler and matrix would affect the degree of contact between filler particles where the interaction (i.e. surface energy) would determine the level of wetting ${ }^{[5]}$. Therefore an increase in the level of polymer coating on each particle would hinder electrical and thermal conduction but improve mechanical strength. The preparation of the mixtures such as processing technique, pressure and temperature would affect both the distribution of the filler and its interaction in the matrix where ideally homogeneous mixtures are desired for predictable properties. The preparation of the composite materials in the previous study were as follows.

Two roll mill - A two roll mill was used to mix the filler and matrix where the front and back rollers were heated to $123{ }^{\circ} \mathrm{C}$ and $128^{\circ} \mathrm{C}$ respectively. Five $300 \mathrm{~g}$ batches were mixed for each composite grade with the aim of filler contents increasing in $10 \mathrm{wt} \%$ increments. The speed of the rollers were varied continuously according to requirement and the melts were manually churned with a mill knife for no less than 30 minutes to ensure good mixing. The sheets of composite produced were then pelletised in a pelletiser.

Ashing test - Ashing tests were done using 10 pellets from each composite grade to find accurate weight percentages after mixing. The oven temperature was set to $500{ }^{\circ} \mathrm{C}$ and total burnout time was 75 min (including ramping) in ambient air. The wt $\%$ of each composite grade was calculated from the pre and post burnout weights of the samples and was repeated twice more for accuracy.

Injection moulding - 15 injection moulded test samples were created for each composite grade as well as the pure polymer with one test sample per shot cycle conforming to ASTM 
D638 Type I test bars. The injected flow direction was parallel to the length of the test samples where the barrel, nozzle and sprue temperatures were $190^{\circ} \mathrm{C}, 200^{\circ} \mathrm{C}$ and $250^{\circ} \mathrm{C}$ respectively. The mould was kept at a constant $30^{\circ} \mathrm{C}$ and the shot was injected at a pressure of $100 \mathrm{bar}$ with a holding pressure of $10 \mathrm{bar}$.

Compression moulding - 15 compression moulded samples for each composite grade and pure polymer were created using a square 3 -plate mould with a $250 \mathrm{~mm}$ x $250 \mathrm{~mm}$ by $3 \mathrm{~mm}$ cavity. Two hot presses were used, one for heating and one for cooling. For carbon black and graphite composites, the heated press was initially set to $170^{\circ} \mathrm{C}$ for the lowest composite filler content and then increased by $15^{\circ} \mathrm{C}$ for every $10 \mathrm{wt} \%$ increase to allow the material to flow sufficiently. The pure polymer samples were formed at $160^{\circ} \mathrm{C}$ and the magnetite filled composites flowed sufficiently at a constant $170^{\circ} \mathrm{C}$ for all composite grades as the filler volume fraction was low due to magnetite's higher density of $5.02 \mathrm{gcm}^{-3}$ compared to $1.82 \mathrm{gcm}^{-3}$ and $2.24 \mathrm{gcm}^{-3}$ for carbon black and graphite respectively. All mouldings were heated for 15 minutes without compression, then compressed at 20tonnes for a further 10 minutes and then cooled for 10 minutes in a water cooled hot press. ASTM D638 Type I test bars were cut from the moulded plates using a $150 \mathrm{~W}$ coherent diamond carbon dioxide pulsed laser.

There are many models in the literature that model electrical, thermal and mechanical properties. Only a few are discussed here where models have been applied to similar composites as the ones presented in the previous study. A brief overview of the common theories behind the types of models used in the literature and the particular models chosen and applied to the experimental results will be given in this paper. A spherical mono dispersed filler phase is assumed for all models due to the spherical particle shape of the 
carbon filler and the boulder-like graphite and magnetite filler shapes coupled with the random orientation observed from both magnetite and graphite micrographs presented previously ${ }^{[1]}$.

\section{Model Theories}

\section{Electrical / Thermal}

As electrical and thermal conductive properties require sufficient particle to particle contact, theories based on this have been developed. The main theory, called percolation theory, has been used to describe the electrical and thermal properties of many materials and has been applied many times to composites like those studied here. It states that near $60 \%$ a critical point is found, called the percolation threshold it is the point at which the composite becomes conductive due to the clusters forming a large continuous network. The percolation threshold in relation to filler content as a volume fraction is illustrated in figure 1.

[Insert Figure 1.]

Figure 1 - Electrical conductivity profile for composites with increasing filler volume fraction displaying different cluster formations. ${ }^{[6]}$

The composite remains non-conductive up to the percolation threshold, at the percolation threshold a conductive network is formed and the volume fraction at that point can be considered the critical volume fraction $\left(\phi_{c}\right)$. The conductivity of the composite increases 
rapidly as the conductive network grows with increasing volume fraction and then starts to level off as the further growth of clusters has little effect on the overall conductivity.

Different fillers have different degrees of conductivity; however within the same material the physical properties of the filler such as size and shape can affect the percolation threshold ${ }^{[7]}$. Fillers that are non-spherical are subject to orientation during processing such as injection moulding and to some extent compression moulding that tend to produce materials with anisotropic conductivity ${ }^{[8,9]}$.

Chemical properties such as the surface energies of the matrix and filler have a role to play in electrical/thermal conductivity. Filler dispersion within the matrix increases as the difference between filler and matrix surface energy decreases. This has an effect of increasing percolation threshold but improves overall conductivity ${ }^{[4]}$.

\section{Mechanical}

There are many theories / computational techniques that are applicable to mechanical property modelling such as mean-field theory, shear lag theory, finite element methods (FEM) and the rule of mixtures.

- Mean field theory (or self consistent field theory) aims to reduce a many-body system that is generally very difficult to solve with all its interactions to a one-body problem with an average or effective interaction. 
1

2

3

4

5

6

- Shear-lag theory explains the transfer of load into a fibre from the surrounding matrix and therefore is useful for fibre loaded materials.

- FEM is a computational technique for finding approximate solutions of partial differential equations by either eliminating or approximating the partial differential equations into ordinary differential equations to simplify calculations.

- The rule of mixtures is a simplified model that estimates mechanical behaviour reasonably well. Compared to the other models / theories mentioned, the rule of mixtures is very basic in its approach and is the foundation for more complex models that can improve upon its predictive abilities.

The rule of mixtures can be used to predict the elastic modulus of composites from the elastic moduli and volume fractions of the filler particles and the matrix ${ }^{[10]}$. It states that the modulus of a unidirectional fibre composite is proportional to the volume fractions of the materials in the composite. Elastic modulus of the composite is estimated by the sum of the two products derived by multiplying the elastic modulus of the reinforcement material and the matrix material by their respective volume fractions ${ }^{[10]}$ and is given by:

$$
E_{c}=E_{f} V_{f}+E_{m}\left(1-V_{f}\right)
$$

Equation 1 is known as the Voigt model, also called the upper bound of the rule of mixtures. Later, Reuss and Angew ${ }^{[11]}$ described a uniform stress model, referred to as the lower bound of the rule of mixtures, given by:

(Equ 2.) 


$$
E_{c}=\left[V_{f} / E_{f}+\left(1-V_{f}\right) E_{m}\right]^{-1}
$$

The rule of mixtures can be useful to estimate elastic modulus but it is not an accurate numerical method for modeling. Models/theories based on the rule of mixtures that are more accurate predictors of mechanical behaviour are explained in the next section.

\section{Models}

\section{Electrical Conductivity}

Three models compared by Clingerman et al ${ }^{[12]}$ are statistical, thermodynamic and structure oriented models. All the models take into account the volume fraction of the filler, however not all require known conductivities of the filler or polymer. The volume fraction should be known to account for dispersion levels and the proximity between filler particles to gauge the degree of contact between them.

Mclachlan's (statistical) model proposed for conductive particles in an insulating matrix takes into account the resistivities of the constituents $(\rho)$, volume fraction $(\phi)$, the percolation threshold $\left(\phi_{\mathrm{c}}\right)$ and a critical exponent $(\mathrm{t})$. The critical exponent can be found by calculation and is a function of the demagnitization or depolarization coefficients of the low and high resistivity materials or it can be found by curve fitting ${ }^{[12]}$.

$$
\frac{(1-\phi)\left(\rho_{m}^{1 / t}-\rho_{h}^{1 / t}\right)}{\rho_{m}^{1 / t}+\left(\frac{1-\phi_{c}}{\phi_{c}}\right) \rho_{h}^{1 / t}}+\frac{\phi\left(\rho_{m}^{1 / t}-\rho_{l}^{1 / t}\right)}{\rho_{m}^{1 / t}+\left(\frac{1-\phi_{c}}{\phi_{c}}\right) \rho_{l}^{1 / t}}=0
$$


The model, although considered an accurate statistical model, can be difficult to calculate (for the critical exponent) if the demagnitization and depolarization coefficients are unknown. The critical exponent would then have to be found by curve fitting which was found unsuccessful according to Clingerman et al ${ }^{[12]}$.

The Lewis-Neilsen Model (equation 4) which is a structure oriented percolation model, relates the conductivity to the aspect ratio and the co-ordination number of the filler (packing factor F), where constant A is fixed for a particular particle's morphology ${ }^{[13]}$.

$$
\begin{gathered}
\sigma_{m}=\sigma_{l}\left(\frac{1+A B \phi}{1-B \Psi \phi}\right) \\
B=\frac{\sigma_{h} / \sigma_{l}-1}{\sigma_{h} / \sigma_{l}+A} \quad \Psi \approx 1+\left(\frac{1-F}{F^{2}}\right) \phi
\end{gathered}
$$

Structure orientated models do not take into account or predict surface energy interactions between the filler and matrix which has been considered a major limitation with this classification of model ${ }^{[14]}$. Also the model requires the conductivity of the filler $\left(\sigma_{\mathrm{h}}\right)$ which could not be obtained in this investigation.

One thermodynamic model by Mamunya ${ }^{[15]}$ exists which takes into account the interactions between the polymer and filler using interfacial tension and surface energies in addition to the size and quantity of material where a packing factor of the filler takes into account filler morphology. However, the model predicts the rapid increase in conductivity (i.e. the linear portion of the conductivity profile) to the levelled off portion of the conductivity profile near 
to the maximum filler conductivity as can be seen in figure 1. Another similar model by Mamunya ${ }^{[15,16]}$, which does not take into account surface energy interaction of the filler and matrix, can be used to model electrical conductivity behaviour based on a critical exponent. It can be used to predict the electrical conductivity from the percolation threshold up to the somewhat linear portion of the conductivity profile and is given by the equation:

$$
\sigma=\sigma_{c}+\left(\sigma_{m}-\sigma_{c}\right) \cdot\left(\frac{\phi-\phi_{c}}{F-\phi_{c}}\right)^{t} \quad F=\frac{V_{f}}{V_{f}+V_{p}}
$$

According to Clingerman et al, Mamunya's model provided the best fit to experimental data $[12,14]$. For this study the Mamunya model was thought to offer the most accurate prediction of electrical conductivity with little input from experimental data as well as the fact that the electrical conductivity of the fillers could not be obtained. For optimum fitting of the model to the electrical conductivity data using the critical exponent $t$, a least squares fit method was employed.

\section{Thermal Conductivity}

As the requirements for thermal conductivity are similar to electrical conductivity, the electrical conductivity models are still relevant. McLachlan's model exchanges electrical resistivity parameters with thermal resistivities and the Lewis-Nielsen model replaces electrical conductivity parameters with thermal conductivities. The Mamunya thermal conductivity model differs slightly from the electrical model and has been used to explain the steeper rise in thermal conductivity with increasing volume fraction ${ }^{[17]}$.

$$
\log \lambda=\log \lambda_{\mathrm{p}}+\left(\log \lambda_{\mathrm{f}}-\log \lambda_{\mathrm{p}}\right)(\phi / F)^{\mathrm{N}}
$$


The model proposes that the ratio of filler to matrix thermal conductivity is not more than $10^{3}$ orders of magnitude whereas it can be $10^{10}-10^{20}$ orders of magnitude for electrical conductivity. A percolation threshold only appears at conductivity ratios above $10^{5}$ and have applied only to systems with conductive sites within a non-conductive medium ${ }^{[17]}$.

For thermal conductivity the Mamunya model was chosen based on similar reasoning to the electrical models where McLachlan's critical exponent would be difficult to incorporate if unknown. As the Lewis-Nielsen model has been shown to incur a major limitation with the prediction of electrical conductivity with regards to surface energy, it was unstated as to whether similar inaccuracies would transfer to thermal conductivity. As stated before, the main reason for excluding this model was the use of filler conductivity in the calculations.

\footnotetext{
Mamunya stated that previous modelling work ${ }^{[18-21]}$ showed an absence of percolation behaviour, the lack of thermal percolation threshold is due to the thermal conduction properties of the matrix where the polymer will have its own thermal conductivity regardless of filler content. Also previous work conducted to account for the additional thermal conductivity ${ }^{[22,23]}$ did not take into account a realistic filler geometry connected to the packing factor. Therefore it was thought that the current Mamunya model, as a result of previous investigations, would give better results and account for more of the filler parameters and processing through the use of the exponent $\mathrm{N}$ just like the exponent $\mathrm{t}$ for electrical conductivity.
} 
For dispersed fillers the packing factor $(\mathrm{F})$ still changes from 0 to 1 however in this study $\mathrm{F}$ is always less than 1 . If $\mathrm{F}=1$ and $\mathrm{N}=1$ where a continuous second phase is present in the matrix then equation 6 reduces back to the Lichtenecker equation.

In keeping with the data analysis conducted by Mamunya et al [16, 17], the thermal conductivity $(\lambda)$ models were plotted together with the experimental data on a $\log \lambda$ vs filler volume fraction graph. This was done so that thermal conductivity behaviour according to the exponent $\mathrm{N}$ in the thermal conductivity model, could be analysed where deviations from $\mathrm{N}=1$ (for linear relationships) results in a change in local filler concentration in the structure. The model fitting to the thermal conductivity data was done using a least squares fit to gain optimum values for $\mathrm{N}$.

\section{Mechanical Properties}

As mentioned previously, the rule of mixtures can provide accurate predictions when the differences between elastic moduli of the two phases are small but generally is used only as an estimate for elastic modulus ${ }^{[10]}$. More accurate forms of the rule of mixtures for prediction of mechanical behaviour are Hirsch's model, Guth's model and Kerner's equation. The models are elastic and flexural modulus models that are well known for predicting mechanical properties of composites. They do not account for non-spherical particle shapes as a spherical mono dispersed filler phase is assumed for all models.

Hirsch's empirical model uses a combination of equal strain and equal stress conditions in the filler and matrix phases ${ }^{[24]}$ and is given by: 


$$
E_{c}=x\left(E_{f} V_{f}+E_{m} V_{m}\right)+(1-x)\left[\left(E_{f} E_{m}\right) /\left(E_{f} V_{m}+E_{m} V_{f}\right)\right]
$$

\begin{abstract}
An empirical factor (x) is obtained from experimental data and varies between 0 for isostress and 1 for isostrain. Hirsch's model is an accurate predictor of modulus as proven by Shyang et al ${ }^{[25]}$ and Kalaprasad et al ${ }^{[26]}$ by comparison with numerous other models. However it is an empirical model and is reliant on experimental data such as the elastic modulus of the filler $\left(E_{f}\right)$ that would be difficult to obtain for powdered materials.
\end{abstract}

Guth's model for spherical particles shown in equation 8 is reliant on an Einstein Coefficient $\left(\mathrm{K}_{\mathrm{E}}\right)$ which is an important factor in the equation that can be calculated by the Poisson's ratio of the matrix and the relative Einstein coefficient ratio where $\mathrm{K}_{\mathrm{E}}=2.5$ for a material with a Poisson's ratio of $0.5^{[13,27,28]}$.

$$
E_{c}=E_{m}\left(1+K_{E} V_{f}+14.1 V_{f}^{2}\right)
$$

Guth's model significantly over predicted the results along with the rule of mixtures as described in the work of Shyang et al ${ }^{[25]}$. Shyang et al used spherical hydroxyapatite (HA) particles as reinforcement in a polymethyl methacrylate (PMMA) matrix. They found that Hirsch's model came closest to the experimental results and Kerner's equation was slightly less accurate than Hirsch's model.

The study of spherical HA particles in PMMA was thought to be closely related to the aims of this study of conductive fillers in polyethylene for mechanical properties. With this in mind, Kerner's equation was chosen to model the elastic and flexural moduli of the composites used. Kerner's equation assumes adhesion between the filler and matrix and so may not do well for fillers with compatibility problems in polymer matrices ${ }^{[25]}$. The model 
is useful for composites containing randomly dispersed spherical or nearly spherical filler

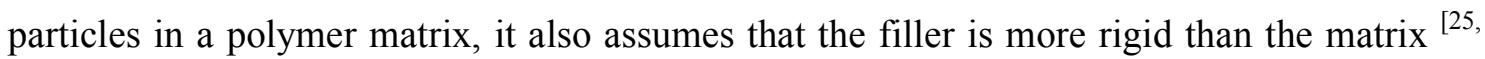
29]. Although Hirsch's model would be more accurate, Kerner's equation would be less reliant on experimental data for parameters used in the model.

Kerner's equation ${ }^{[29]}$ (equation 9 ) can be used for any modulus ${ }^{[30]}$ where the parameter $\mathrm{E}_{\mathrm{m}}$ (modulus of the matrix) represents the required modulus of the user.

$$
E_{c}=E_{m} \cdot 1+\left\{\left[15\left(1-v_{m}\right) V_{f}\right] /\left(8-10 v_{m} V_{m}\right)\right\}
$$

Initially the models for elastic and flexural modulus were plotted using inputs for the elastic and flexural modulus of the pure polyethylene, however the equation did not take into account different fillers within the same type of matrix where variations in magnitude of the modulus would occur with different types of filler. Variations in the results with different fillers would originate from filler properties such as size, shape and surface energy (compatibility with the matrix). Plots using the Kerner's equation gave shallow increases in modulus with increasing filler content and did not fit the data due to the large step increase in moduli with the initial addition of fillers.

Therefore Kerner's equation was adapted where the initial volume fraction of the filler $\left(\mathrm{V}_{\mathrm{f}}\right)$ is no longer zero and the data points for the polymer elastic and flexural moduli are ignored. The initial volume fraction of each plot is equal to the first set of experimental data gained, i.e. the lowest filler content in the data set and the elastic and flexural modulus $\left(\mathrm{E}_{\mathrm{m}}\right)$ of the matrix is the modulus value at the lowest filler content. If the lowest filler volume fraction in 
the data set is $\mathrm{V}_{\mathrm{f}}$, and the new (matrix) elastic/flexural modulus is $\mathrm{E}_{\mathrm{m}}$ ' then the following equation is procured.

$$
E_{c}=E_{m}{ }^{\prime}\left[1+\left\{\left[15\left(1-v_{m}\right) V_{f}-V_{f}^{\prime}\right] /\left(8-10 v_{m}\left(1-V_{f}-V_{f}^{\prime}\right)\right)\right\}\right]
$$

The adapted equation sets the origin of the plot at the lowest filler volume fraction and any increases in filler volume fraction are relative to the new origin. Therefore if $\mathrm{V}_{\mathrm{f}}{ }^{\prime}=0.1$ and the next data point is at $V_{f}=0.25$ then the increase in volume fraction computed by the equation is 0.15 . The models were plotted with modulus $\left(E_{c}\right)$ vs. the original volume fraction $\left(\mathrm{V}_{\mathrm{f}}\right)$ that has been converted to weight percentage. Data for pure polyethylene has also been plotted for comparison but is not part of the model fitting.

\section{Results \& Discussion}

The least squares regression method used to gain optimum fitting of the electrical and thermal conductivity models via critical exponents was done using the formula in equation 11 . The least squares fit was also used to judge model accuracy for the elastic and flexural moduli data.

$$
\begin{gathered}
R^{2}=1-\frac{S S_{r e s}}{S S_{\text {tot }}} \\
S S_{r e s}=\sum_{i}\left(x_{i}-f_{i}\right)^{2} \quad S S_{\text {tot }}=\sum_{i}\left(x_{i}-\bar{x}\right)^{2}
\end{gathered}
$$

\section{Electrical Conductivity}


As stated in the previous study ${ }^{[1]}$ there were no electrical conductivity data obtained for compression moulded carbon black samples and injection/compression moulded magnetite samples due to moulding problems and no measurable conductivity respectively. Due to disparities in results that arise from alternative processing techniques it was thought that a fair comparison could not be done using data from other sources. Mamunya's electrical conductivity model was applied to the injection moulded carbon black composites and the injection/compression moulded graphite composites. As there were only three experimental data points for the modelling where the minimum and maximum conductivities and volume fractions are used in the model equation, the only point used to fit the model to the experimental data was the remaining data point at 56 and $57 \mathrm{wt} \%$ for carbon black and graphite respectively.

\begin{abstract}
Although the model used volume fraction in its calculation the results were plotted against weight percentage to allow easy comparison with the previous study as shown in figure 2 . In addition, since the in plane and through plane conductivities were similar and isotropic filler particles were assumed the in plane and through plane conductivities were averaged where the scatter bars show the maximums and minimums from a combined data set.
\end{abstract}

[Insert Figure 2.]

Figure 2 - Model fitting of electrical conductivity of injection moulded carbon black and injection / compression moulded graphite composites. 
Values for the critical exponent (t) at 56 and $57 \mathrm{wt} \%$ were found to be 2.31 for the injection moulded carbon black composite and 2.98 and 5.69 for the injection and compression moulded graphite composites respectively. According to percolation theory the value of $t$ is in the range of 1.6-1.9 ${ }^{[6]}$. In the work of Mamunya et al it was stated that the percolation theory with $\mathrm{t}$ values of 2.4 to 3.2 still hold true for systems with dispersed random filler distributions. The accepted universal value for $\mathrm{t}\left(\mathrm{t}_{\mathrm{un}}\right)$ is $1.7^{[31]}$, any increase in $\mathrm{t}$ is denoted by $\mathrm{t}=\mathrm{t}_{\mathrm{un}}+\mathrm{t}_{\mathrm{add}}$ where $\mathrm{t}$ additional ( $\mathrm{t}_{\mathrm{add}}$ ) would infer the conductivity contribution of different mechanisms. Mechanisms such as tunnelling conductivity and complex conductive cluster structures with the presence of nodes and blobs ${ }^{[17]}$.

Further models have been used to calculate $\mathrm{t}$ based on filler contact patterns such as Balberg's model for tunnelling conductivity ${ }^{[32]}$ and the Links-nodes-blobs (LNB) model ${ }^{[33]}$. Balberg gave a $\mathrm{t}$ value of 2.8 for Cabot-black compared to a Ketjen-black $\mathrm{t}$ value of 2.0 where the tunnelling conductance $g$ was determined by the complexity of the structure of the carbon aggregates. Defined as high and low structures, the higher $\mathrm{t}$ value would denote higher complexity in the aggregate structure and lower $\mathrm{t}$ value for lower structure complexity as shown in figure 3.

[Insert Figure 3.]

Figure 3 - Balberg's Structures ${ }^{[32]}$ - (a) No structure, (b) low structure, (c) high structure.

The LNB model that is applicable to t values greater than 2 describes conductivity through link, node and blob structures. Links are lengths of single bonds which if cut will interrupt the current flow, nodes are sites that are connected to the boundaries by at least three 
independent paths and blobs are multiple connected paths on a backbone where each path carries a fraction of the backbone current. These structures are shown in figure 4 .

[Insert Figure 4.]

Figure 4 - Links Nodes and Blob structures ${ }^{[33]}$ - (a) Nodes, (b) Links, (c) Blobs. $\xi$ is a unit length.

As with Balberg's model, the higher the $\mathrm{t}$ value the more complex the structure. Unknown proportions of the contributions from tunnelling and LNB is the most likely outcome of the conductivity mechanisms represented by the critical exponents. The very high value of the compression moulded graphite was comparable to the work of Celzard et al ${ }^{[34]}$ where high values of 7 (in plane) and 10 (through plane) for the critical exponents of their anisotropic graphite composite have been observed. Celzard et al concluded that the particles were more or less covered by a thin layer of polymer hindering direct particle contact and therefore conduction would occur via tunnelling. They reasoned that the conductivity in the highly loaded samples were several orders of magnitude less than the basal plane conductivity of the graphite powder so the high interparticle resistances must have been due to the thin polymer layers.

\section{Thermal Conductivity}

The thermal conductivity modelling results of the injection and compression moulded composites shown in figure 5 were fitted using the least squares fit method. 


\title{
[Insert Figure 5.]
}

Figure 5 - Thermal conductivity model fittings to polyethylene carbon black, graphite and magnetite composite data for injection and compression moulding.

\begin{abstract}
The model fitting for the carbon black and graphite composites showed good fits with $\mathrm{R}^{2}$ values above 0.95 . The exponents $(\mathrm{N})$ gained from the fitting varied above and below 1 , when $\mathrm{N}$ deviates positively from 1 the structure has displayed a more dense packing of the filler resulting in an increased local filler concentration ${ }^{[17]}$ thus higher conductivity. It can be inferred that a negative deviation from $\mathrm{N}=1$ suggests that less packing of the filler has resulted in a lower local filler concentration. The magnetite composites showed slightly reduced fits with $\mathrm{R}^{2}$ values between 0.93 and 0.94 , the fitting generated a hyperbolic curve where the rate of increase in thermal conductivity decreased with increasing magnetite content and represented reduced local filler concentrations.
\end{abstract}

\section{Elastic / Flexural Modulus}

[Insert Figure 6.]

\begin{abstract}
Figure 6 - Elastic modulus model fittings to polyethylene carbon black, graphite and magnetite composite data for injection and compression moulding.
\end{abstract}

The injection moulded models fitted the experimental data very well giving $\mathrm{R}^{2}$ values not less than 0.96 however the compression moulded models over predicted and did not fit the data very well with no $\mathrm{R}^{2}$ values gained for carbon black and graphite composites and a low $\mathrm{R}^{2}$ 
value of 0.745 for magnetite composites. According to Speake et al ${ }^{[35]}$, the experimental data points should lie lower than the model if there was poor stress transfer from matrix to filler as Kerners equation assumes continuous stress transfer across the filler-matrix boundary. The reduction in polymer at high filler contents, ie reduction in filler-matrix interfaces, would produce poor stress transfer and have a negative effect on the modulus where the data would be expected to deviate from the model. Due to the high density of magnetite the resulting filler volume fraction is far lower with a higher matrix content than that for carbon black and graphite composites at the same wt\%. The plot displayed by the compression moulded magnetite data set could indicate inhomogeiety arising from compression moulding.

[Insert Figure 7.]

Figure 7 - Flexural modulus model fittings to polyethylene carbon black, graphite and magnetite composite data for injection and compression moulding.

With the exception of the injection moulded graphite model, the flexural modulus models displayed in figure 7 for the injection and compression moulded carbon and graphite composites showed poor agreement with the experimental data with $\mathrm{R}^{2}$ values no higher than 0.526. In both, injection more than compression, the models under predicted with shallower gradients than the experimental data. For the carbon black compression moulded samples it is unclear as to whether the data would have deviated further from the model due to the limited number of data points resulting from processing problems ${ }^{[1]}$. The model may not account for differences in test speed and processing, slow test speeds could transfer greater loads to the specimen before fracture resulting in greater modulus than the model predicts and 
processing variations within and between injection and compression moulding may generate similar increases in modulus compared to the model.

\section{Conclusions}

The electrical, thermal and mechanical models chosen for this study have been applied to similar research where spherical particles have been assumed as a filler in an elastic matrix $[12,14,17]$. The Mamunya model for electrical and thermal conductivities were semi-empirical models where the exponents were used to find the best fit. The models showed reasonable agreement with the experimental data where deviations from the models denoted specific composite behaviours.

The optimum exponent values found by tuning for electrical and thermal conductivity models were on the whole greater than those by Mamunya and Clingerman where the ambient processing pressures of Mamunya ${ }^{[15,17]}$ as well as the unspecified holding pressure of Clingerman ${ }^{[14]}$ could have produced low exponents. Therefore the increase in processing pressure could be one contributor to greater exponent values seen in this study due to increases in filler structure complexity with increasing pressure.

Electrical conductivity exponent values greater than the universal value of 1.7 explained that there was a presence of tunnelling conductivity and links, nodes and blobs (LNB) ${ }^{[32,33]}$. These alternative conduction mechanisms due to increased filler structure complexity have contributed to the sharp increases in electrical conductivity observed. However the degree of filler structure complexity and tunnelling conductivity cannot be determined by the Balberg 
and LNB theories ${ }^{[32,33]}$. Similarly the compression moulded graphite composites exhibited an exponent value approaching 7 which was observed by Celzard et al ${ }^{[34]}$ for their anisotropic graphite filler however the level of anisotropy cannot be determined.

The thermal conductivity exponent gained from the fittings showed deviations from 1 where a positive deviation displayed a more dense packing of the filler resulting in an increased local filler concentration thus higher conductivity and negative deviations suggest the opposite $^{[17]}$.

Although the exponents do not provide information on levels of conductivity (maximum and percolation threshold) which would have to be gained experimentally and the magnitude of the various conduction mechanisms cannot be determined, the exponents are useful in gauging relative conductivity behaviour between fillers.

Kerner's equation for elastic and flexural moduli under predicted and only predicted composite behaviour based on the pure polymer properties. A modified Kerner's equation was employed and although the new equation predicted elastic and flexural modulus better than the original equation, positive and negative deviations from the model were observed. According to Speake et al ${ }^{[35]}$, stress transfer across filler matrix interfaces could explain the observations where over predictions of the model would indicate poor stress transfer and under predictions would indicate increased stress transfer than the model would assume.

Overall the modified Kerner's equation predicted elastic modulus better than flexural modulus and so may not be suitable as a universal modulus predictor as the literature claims [30], however more materials with larger data sets should be investigated to confirm this. 
Future study should also investigate adaptations to all models to account for increases in processing pressure and its effect on compressibility of the molten polymer, volume fraction of the matrix and on tunnelling and link, node and blob conduction mechanisms with respect to their occurrence and magnitude.

\section{Funding Acknowledgement}

This research received no specific grant from any funding agency in the public, commercial, or not-for-profit sectors.

\section{Nomenclature}

A - Function of aspect ratio and orientation

B - Constant, equal to 1 for polymer systems

$E_{c}$ - Elastic/Flexural modulus of the composite.

$E_{f}$ - Elastic/Flexural modulus of the filler.

$E_{m} / E_{m}$ ' - Elastic/Flexural modulus of the matrix.

F - Packing factor

$f$ - Modelled data

$\mathrm{K}_{\mathrm{E}}$ - Einstein Coefficient

http://mc.manuscriptcentral.com/(site) 
$\lambda$ - Thermal conductivity

$\lambda_{\mathrm{f}}$ - Thermal conductivity of the filler

$\lambda_{p}$ - Thermal conductivity of the polymer

$v_{\mathrm{m}}$ - Poisson's ratio of the matrix

$\mathrm{N}$ - Exponent

$\rho_{h}$ - Electrical resistivity of the filler

$\rho_{1}$ - Electrical resistivity of the polymer

$\rho_{m}$ - Electrical resistivity of the composite

$\mathrm{R}^{2}$ - Coefficient of determination

$\sigma / \sigma_{\mathrm{m}}$ - Electrical conductivity of the composite

$\sigma_{c}$ - Electrical conductivity at the percolation threshold

$\sigma_{\mathrm{h}}$ - Electrical conductivity of the filler

$\sigma_{1}$ - Electrical conductivity of the polymer

$\sigma_{\mathrm{m}}$ - Electrical conductivity at the maximum packing factor

$\mathrm{SS}_{\text {res }}$ - Sum of squares of the residuals

$\mathrm{SS}_{\text {tot }}$ - Total sum of squares

$\mathrm{t}$ - Critical exponent

$\mathrm{V}_{\mathrm{f}}$ - Lowest filler volume fraction

$V_{p} / V_{m} /\left(1-V_{f}\right)$ - Volume fraction of matrix.

$\phi / V_{f}$ - Volume fraction of filler.

$\phi_{\mathrm{c}}$ - Percolation threshold

$\mathrm{x}$ - Experimental data

$\bar{x}$ - Mean of the experimental data 


\section{References}

[1] Greenwood P, Thring R. H, Chen R. 2012. Conductive materials for polymeric bipolar plates: Electrical, thermal and mechanical properties of polyethylene-carbon black / graphite / magnetite blends. Proc IMechE part L: Journal of materials: design and applications. Vol. 227, Issue. 3, pp. 226-242.

[2] Lux F. 1993. Models proposed to explain the electrical conductivity of mixtures made of conductive and insulating materials. Journal of Materials Science. Vol. 28, pp. 285-301.

[3] Jing, X, Zhao, W, and Lan, L. 2000. The effect of particle size on electric conducting percolation threshold in polymer/conducting particle composites. Journal of Materials Science Letters. Vol. 19, pp. 377-379.

[4] Mamunya E. P, Davidenko V. V and Lebedev E.V. 1997. Effect of polymer-filler interface interactions on percolation conductivity of thermoplastics filled with carbon black. Composites Interfaces. Vol. 4, No 4, pp.169-176.

[5] Mapleston, P. (1992). Conductive Composites Get a Growth Boost from Metallic Fibers, Modern Plastics, 69(May): pp. 80-83.

[6] Stauffer D. 1985. Introduction to percolation theory. London: Taylor \& Francis Ltd. 
[7] Dovzhenko, A.Y, and Bunin V.A, Effect of the Shape and Size of Conducting Particles on the Percolation Cluster Formation in a Ceramic Composition. Technical Physics, Vol. 48, No. 8, 2003, pp. 1058-1060.

[8] Nagata K, Iwabuki H and Nigo H. 1998. Effect of particle size of graphites on electrical conductivity of graphite/polymer composite. Composite Interfaces. Vol. 6, No. 5, pp. 483495.

[9] Webber M. E and Kamal M. R. 1997. Estimation of the volume resistivity of electrically conductive composites. Polymer Composites. Vol. 18, No. 6 pp. 711-725.

[10] Sakaguchi R. L, Wiltbank B. D, Murchison C. F. 2004. Prediction of composite elastic modulus and polymerization shrinkage by computational micromechanics. Dental Materials. Vol. 20, pp. 397-401.

[11] Reuss A, Angew Z. 1929. Berechnung der fliessgrenze von Mishkristallen auf Grund der Plastizitatsbeding fur Einkristalle. Math Mech. Vol.9 pp.49-58.

[12] Clingerman M. L, King J. A, Schulz K. H and Meyers J. D. 2002. Evaluation of electrical conductivity models. Journal of Applied Polymer Science, Vol. 83, pp.13411356.

[13] Pal R. 2008. On the Lewis-Nielsen model for thermal/electrical conductivity of composites. Composites: Part A: Applied Science and Manufacturing. Vol. 39, pp. 718-726. 
[14] Clingerman M. L, Webber E. H, King J. A and Schulz K. H. 2003. Development of an Additive Equation for Predicting the Electrical Conductivity of Carbon- Filled Composites. Journal of Applied Polymer Science, Vol. 88, pp.2280-2299.

[15] Mamunya, E.P, Davydenko V.V, and Lebedev E.V. 1995. Percolation conductivity of polymer composites filled with dispersed conductive filler. Polymer Composites. Vol.16 No.4, pp. 318-324.

[16] Mamunya, Y.P, Muzychenko, Y.V, Pissis, P, Lebedev, E.V, Shut, M.I. 2002. Percolation phenomena in polymers containing dispersed iron. Polymer Engineering science. Vol. 42, No.1, pp. 90-100.

[17] Mamunya Y. P, Davydenko V. V, Pissis P, and Lebedev E.V. 2002. Electrical and thermal conductivity of polymers filled with metal powders. European Polymer Journal. Vol. 38, pp.1887-1897.

[18] Progelhof R.C, Throne J. L and Ruetsch R. R. 1976. Methods for predicting the thermal conductivity of composite systems: A review. Polymer Engineering and Science. Vol. 16, pp. 615-625.

[19] Kusy R. P and Corneliussen R.D. 1975. The thermal conductivity of nickel and copper dispersed in poly(vinyl chloride). Polymer Engineering and Science. Vol.15, pp. 107112. 
[20] Bigg D.M. 1995. Thermal conductivity of heterophase polymer compositions. Advances in Polymer Science. Vol. 119, pp. 1-30.

[21] Sundstrom D. W and Lee Y.D. 1972. Thermal conductivity of polymers filled with particulate solids. Journal of Applied Polymer Science. Vol. 16, pp. 3159-3167.

[22] Agari Y and Uno T. 1985. Thermal Conductivity of Polymer Filled with carbon materials: Effect of Conductive Particle Chains on Thermal Conductivity. Journal of Applied Polymer Science. Vol. 30, pp. 2225-2235.

[23] Rymarenko N. L, Voitenko A.I, Novikov V.V and Privalko V.P. 1992. Melting and Non-isothermal Crystallization of Highly Filler Polymers. Ukrainian Polymer Journal. Vol. 1, p. 259-266.

[24] Karam G. N. 1994. Effect of Fiber-Fiber Interaction on the Strength Properties of Short Fiber Reinforced Cements. Journal of Composites Technology \& Research. Vol. 16 pp. $154-160$.

[25] Shyang C. W, Khim L. Y, Ariffin A, Arifin Z and Ishak M. 2008. Flexural Properties of Hydroxyapatite Reinforced Poly(Methyl Methacrylate) Composites. Journal of Reinforced Plastics and Composites. Vol. 27, pp. 945-952.

[26] Kalaprasad G, Joseph K, Thomas S and Pavithran C. 1997. Theoretical Modeling of Tensile Properties of Short Sisal Fiber-Reinforced Low-Density Polyethylene Composites. Journal of Materials Science. Vol. 32, pp. 4261-4267. 
[27] Yan W, Lin R. J T and Bhattacharyya D. 2006. Particulate reinforced rotationally moulded polyethylene composites - Mixing methods and mechanical properties Composites Science and Technology. 66, pp.2080-2088.

[28] Laven J and Stein H. N. 1991. The Einstein coefficient of suspensions in generalized Newtonian liquids Journal of Rheology. Vol.35, No. 8, pp.1523-1549.

[29] Alger, M.S.M, 1997. $2^{\text {nd }}$ Edition. Polymer Science Dictionary. London: Chapman \& Hall.

[30] Dufresne, A, Cavaille, JY, and Helbert, W. 1996. New Nanocomposite Materials Microcrystalline Starch Reinforced Thermoplastic. Macromolecules. Vol. 29, pp. 7624-7626.

[31] Wu, J, Mclachlan, D.S. Percolation exponents and thresholds obtained from the nearly ideal continuum percolation system graphite-boron nitride. Physical Review B. 1997. Vol. 56, pp.1236-1248.

[32] Balberg I. 1987. Tunneling and nonuniversal conductivity in composite materials. The American Physical Society, Physical Review Letters. Vol. 59, No.12, pp.1305-1308.

[33] Coniglio, A. 1982. Cluster structure near the percolation threshold. Journal of Physics A. Vol. 15. pp.3829-3844. 
[34] Celzard, A, Furdin, G, Marêché, J.F, McRae, E, Dufort, M, and Deleuze, C. 1994. Anisotropic percolation in an epoxy - Graphite disc composite. Solid State Communications. Vol. 92. pp.377-383.

[35] Speake J.H.; Arridge R.G.C.; Curtis G.J. 1974. Measurement of the cure of resins by ultrasonic techniques. Journal of Physics D: Applied Physics. Vol. 7, pp.412-424. 


\section{List of Figures}

Figure 1 - Electrical conductivity profile for composites with increasing filler volume fraction displaying different cluster formations. ${ }^{[6]}$

Figure 2 - Model fitting of electrical conductivity of injection moulded carbon black and injection / compression moulded graphite composites.

Figure 3 - Balberg's Structures ${ }^{[32]}$ - (a) No structure, (b) low structure, (c) high structure.

Figure 4 - Links Nodes and Blob structures ${ }^{[33]}$ - (a) Nodes, (b) Links, (c) Blobs. $\xi$ is a unit length.

Figure 5 - Thermal conductivity model fittings to polyethylene carbon black, graphite and magnetite composite data for injection and compression moulding.

Figure 6 - Elastic modulus model fittings to polyethylene carbon black, graphite and magnetite composite data for injection and compression moulding.

Figure 7 - Flexural modulus model fittings to polyethylene carbon black, graphite and magnetite composite data for injection and compression moulding. 


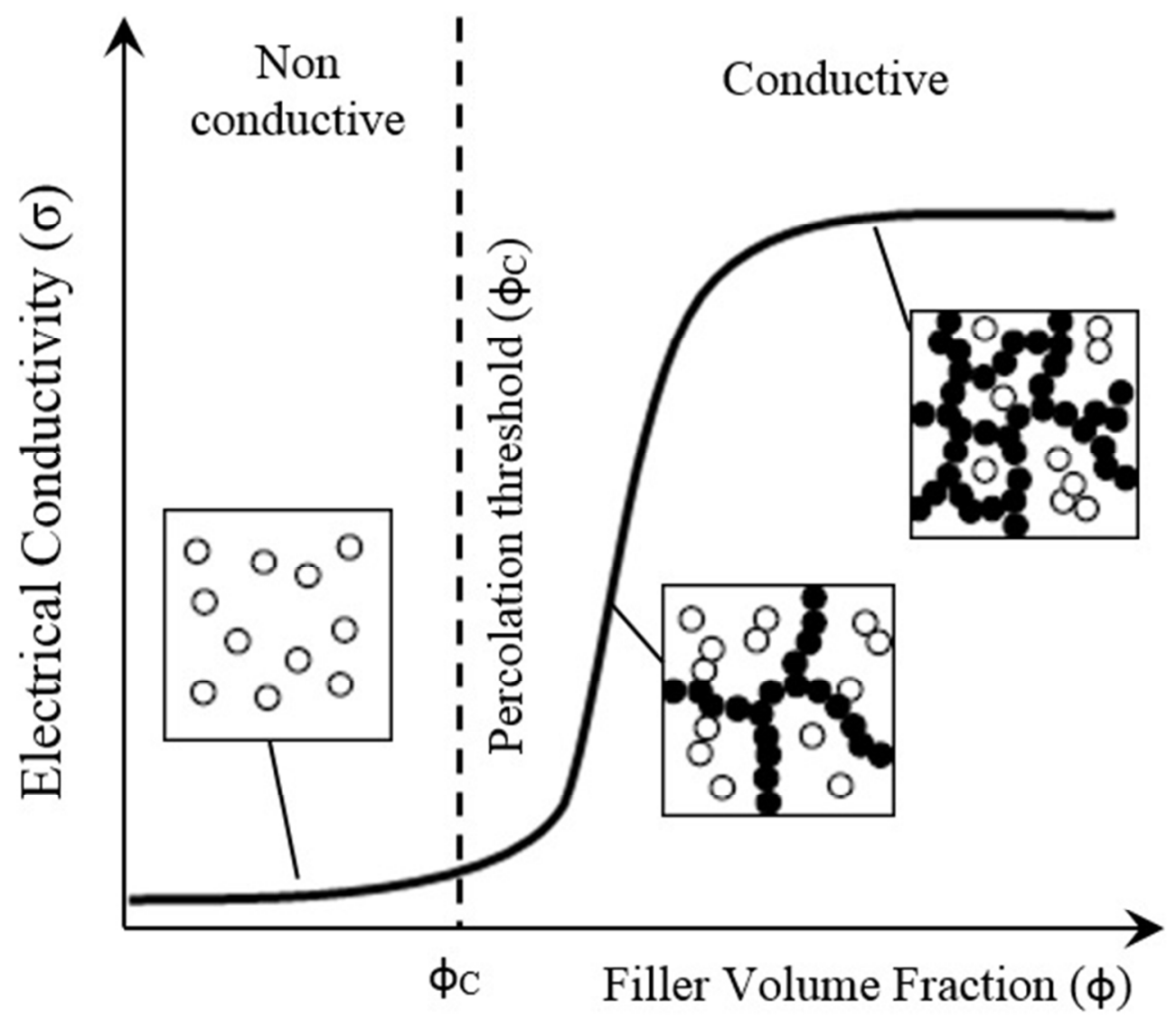

Figure 1 - Electrical conductivity profile for composites with increasing filler volume fraction displaying different cluster formations.

$151 \times 132 \mathrm{~mm}(96 \times 96 \mathrm{DPI})$ 
Figure 2 - Model fitting of electrical conductivity of injection moulded carbon black and injection / compression moulded graphite composites. $227 \times 128 \mathrm{~mm}(96 \times 96 \mathrm{DPI})$ 


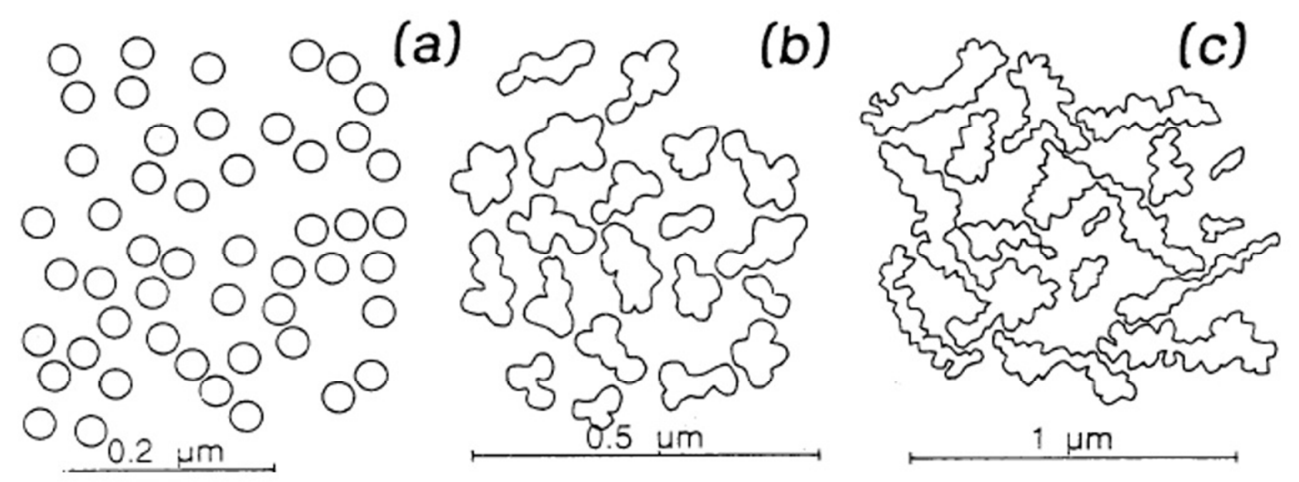

Figure 3 - Balberg's Structures - (a) No structure, (b) low structure, (c) high structure. $275 \times 106 \mathrm{~mm}(96 \times 96 \mathrm{DPI})$ 

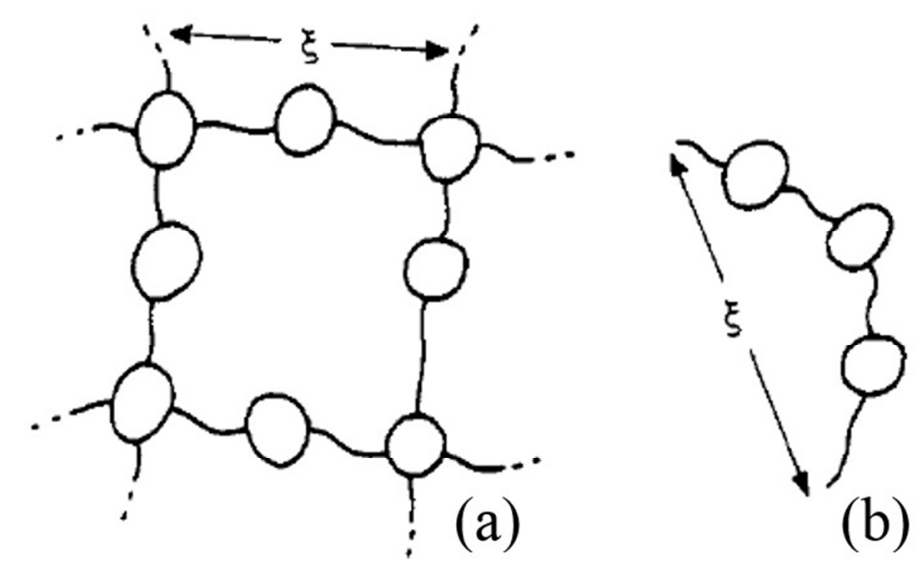

(b)

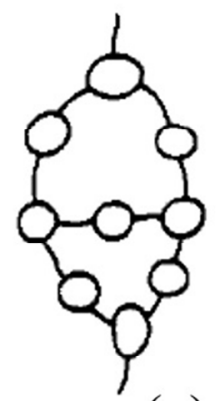

(c)

Figure 4 - Links Nodes and Blob structures - (a) Nodes, (b) Links, (c) Blobs. $\xi$ is a unit length. $272 \times 118 \mathrm{~mm}$ (96 x 96 DPI) 
Thermal Conductivity Models for Injection and Compression Moulded Carbon Black, Graphite and Magnetite Composites

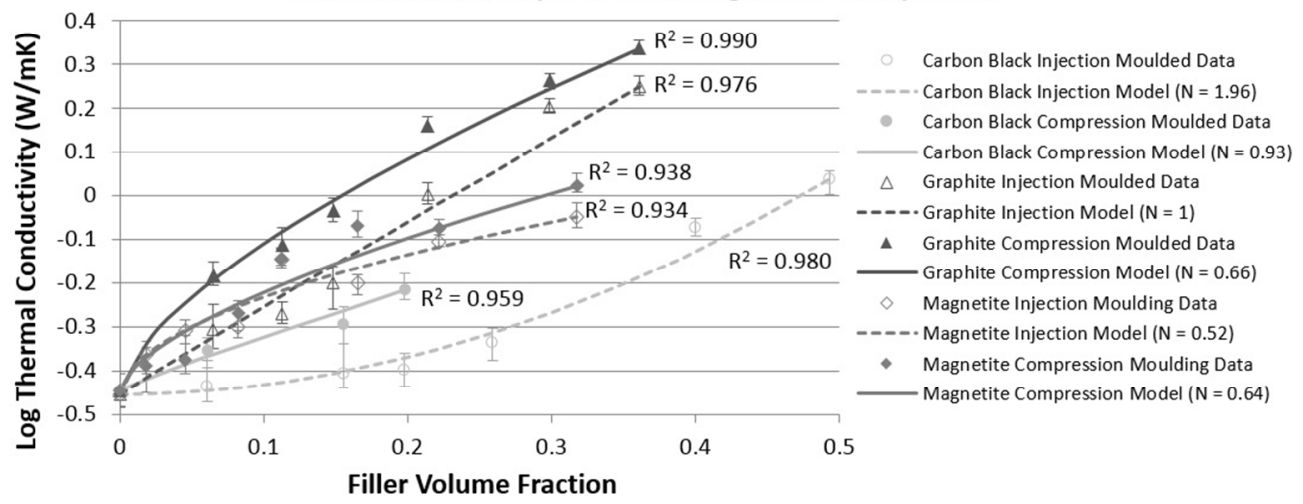

Figure 5 - Thermal conductivity model fittings to polyethylene carbon black, graphite and magnetite composite data for injection and compression moulding. $289 \times 130 \mathrm{~mm}(96 \times 96 \mathrm{DPI})$ 
Figure 6 - Elastic modulus model fittings to polyethylene carbon black, graphite and magnetite composite data for injection and compression moulding. $270 \times 169 \mathrm{~mm}(96 \times 96 \mathrm{DPI})$ 


\section{Flexural Modulus Models for Injection \& Compression} Moulded Carbon Black, Graphite Composites

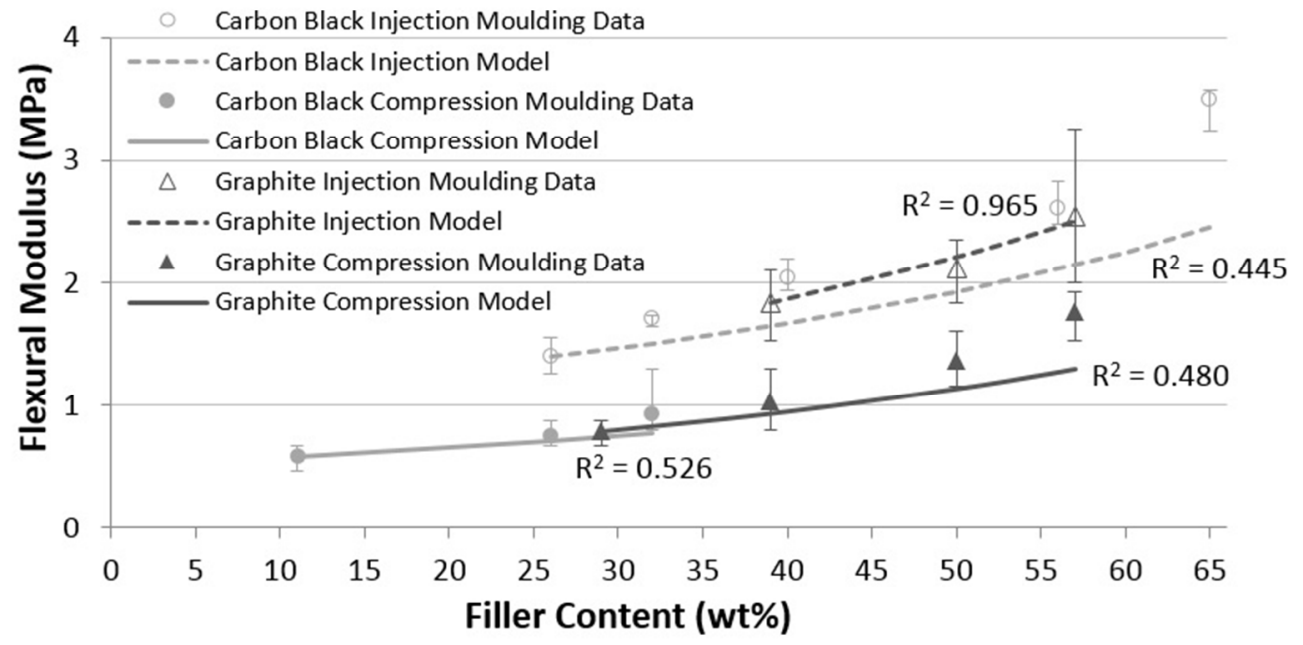

Figure 7 - Flexural modulus model fittings to polyethylene carbon black, graphite and magnetite composite data for injection and compression moulding. $218 \times 129 \mathrm{~mm}(96 \times 96 \mathrm{DPI})$ 